\title{
48 THE USE OF CARDIAC CATHETERISATION IN PRE-OPERATIVE WORK-UP FOR LIVER TRANSPLANTATION
}

C E M McClure, S Ahmed, P W Johnston, W J Cash, N I McDougall Departments of Hepatology and Cardiology, Royal Victoria Hospital, Belfast, County Antrim, Northern Ireland

\subsection{6/gutjnl-2013-305143.48}

Introduction Significant cardiac disease may exclude patients from orthotopic liver transplantation (OLT) or may require intervention to make OLT feasible. Non-invasive methods of cardiac assessment (ECHO and cardiopulmonary exercise stress test $(\mathrm{CPEX})$ ) are used to help minimise the need for cardiac catheterisation (CC).

Aims/Background To audit the use of CC in OLT assessments at a regional liver unit in Northern Ireland.

Method All patients who underwent formal OLT assessment in RVH from January 2008 to June 2012 were included. Information was obtained from the RVH Liver Unit database and cross referenced with radiology and patient centre records. Patient records for all those who had CC were reviewed to determine outcomes.

Results 188 patients underwent full OLT assessment, of whom $43(23 \%)$ required CC. The indications for CC were: 22 had multiple CVS risk factors, 11 had abnormal ECHO, 3 abnormal MPI, 1 abnormal CPEX and 6 other. Overall, 31 (72\%) of 43 CC patients had no significant cardiac disease detected. 12 (28\%) patients had significant cardiac disease, of whom, 7 were not listed and 5 had intervention (3 PCI with stenting, 2 PFO closures (1 not listed)). 27 (62.8\%) patients who underwent CC were listed for OLT. One patient suffered a fatal variceal haemorrhage immediately post CC (without PCI). 


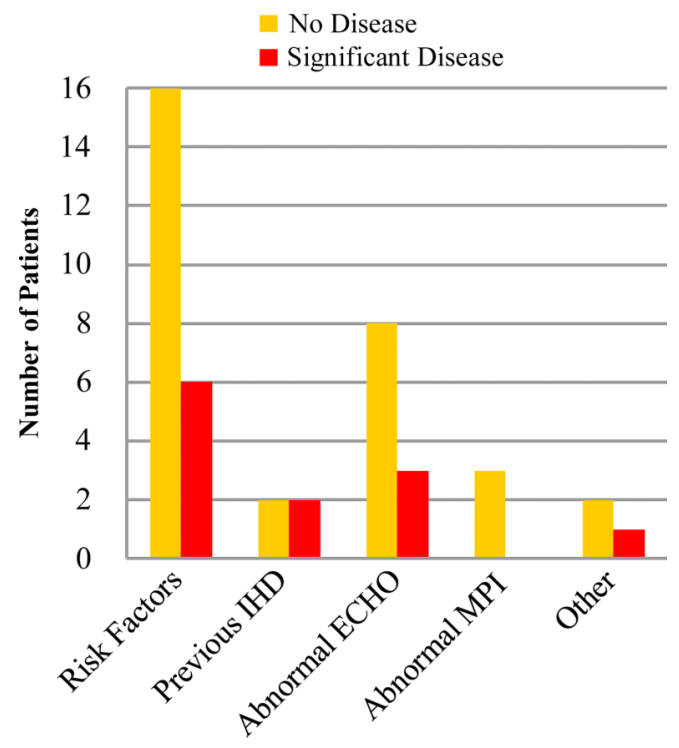

Figure 1 Indications for Cardiac Catheterisation and Results.

Conclusion The most common reasons for requiring CC during OLT workup were multiple CVS risk factors and abnormal ECHO. CPEX rarely detected significant disease. Mortality post CC was $2 \%$, however, interpretation of this result is limited by the small sample size. 